\title{
Solution of Some Integral Equations Involving Confluent $k$-Hypergeometric Functions
}

\author{
Shahid Mubeen \\ Department of Mathematics, University of Sargodha, Sargodha, Pakistan \\ Email:smjhanda@gmail.com
}

Received April 19, 2013; revised May 20, 2013; accepted May 28, 2013

Copyright (C) 2013 Shahid Mubeen. This is an open access article distributed under the Creative Commons Attribution License, which permits unrestricted use, distribution, and reproduction in any medium, provided the original work is properly cited.

\section{ABSTRACT}

The principle aim of this research article is to investigate the properties of $k$-fractional integration introduced and defined by Mubeen and Habibullah [1], and secondly to solve the integral equation of the form $g(x)=\int_{0}^{x} \frac{(x-t)^{\gamma / k}}{\Gamma_{k}(\gamma)}{ }_{1} F_{1}\left[\begin{array}{ll}(\beta, k) ; \\ (\gamma, k) ;\end{array} \quad t-x\right] f(t) \mathrm{d} t$, for $k>0, \beta>0, \gamma>0,0<x<t<\infty$, where ${ }_{1} F_{1}\left[\begin{array}{l}(\beta, k) ; \\ (\gamma, k) ;\end{array}\right]$ is the confluent $k$-hypergeometric functions, by using $k$-fractional integration.

Keywords: Linear Integral Equations; Fractional Integrals; Confluent Hypergeometric Functions

\section{Introduction}

Erdélyi [2] investigated the solutions of integral equations whose kernels contain Legendre functions. Love [3] solved the integral equations involving hypergeometric functions using fractional derivatives. Using variance of fractional integration, Habibullah [4] investigated the solution of the integral equations involving confluent hypergeometric functions and Srivastava [5] discussed the equations with polynomial kernels.

Diaz et al. [6-8] have introduced $k$-gamma and $k$-beta functions and proved a number of their properties that we are interested in. They have also studied $k$-zeta function and $k$-hypergeometric function based on Pochhammer $k$-symbols for factorial functions. These studies were then followed by works of Mansour [9], Kokologiannaki [10], Krasniqi [11,12] and Merovci [13] elaborating and strengthening the scope of $k$-gamma and $k$-beta functions. Very recently, Mubeen and Habibullah [14] gave a simple and useful integral representations of generalized $k$ hypergeometric and confluent $k$-hypergeometric functions that could helpful in completing the present research paper.

\section{Fractional Integration}

Mubeen and Habibullah [1] defined a $k$-fractional integration as a variant of Riemann-Liouville fractional integral as

$$
I_{k}^{\alpha}(f)(x)=\frac{1}{k \Gamma_{k}(\alpha)} \int_{0}^{x}(x-t)^{(\alpha / k)-1} f(t) \mathrm{d} t,
$$

for $k>0,0<x<t<\infty$. It reduces to the classical Riemann-Liouville fractional integral by taking $k \rightarrow 1$ as

$$
I^{\alpha}(f)(x)=\frac{1}{\Gamma(\alpha)} \int_{0}^{x}(x-t)^{\alpha-1} f(t) \mathrm{d} t, 0<x<t<\infty .
$$

\section{3. k-Hypergeometric and Confluent}

\section{$\boldsymbol{k}$-Hypergeometric Differential Equations}

The following $k$-hypergeometric function defined by $\mathrm{Mu}$ been and Habibullah [14]

$$
\begin{aligned}
\omega & ={ }_{2} F_{1, k}\left[\begin{array}{r}
(\alpha, k),(\beta, k) ; \\
(\gamma, k) ;
\end{array}\right] \\
& =\sum_{n=0}^{\infty} \frac{(\alpha)_{n, k}(\beta)_{n, k}}{(\gamma)_{n, k}} \frac{z^{n}}{n !}, k>0
\end{aligned}
$$

is the solution of the linear second order differential equation of the form

$$
k z(1-k z) \omega^{\prime \prime}+[\gamma-k(\alpha+\beta+k) z] \omega^{\prime}-\alpha \beta \omega=0 .
$$

In this article, we call it $k$-hypergeometric differential equation. It reduces to ordinary hypergeometric differential equation by taking $k \rightarrow 1$. 
And also the following confluent $k$-hypergeometric function defined by Mubeen and Habibullah [14]

$$
\omega={ }_{1} F_{1, k}\left[\begin{array}{l}
(\beta, k) ; \\
(\gamma, k) ;
\end{array}\right]=\sum_{n=0}^{\infty} \frac{(\beta)_{n, k}}{(\gamma)_{n, k}} \frac{z^{n}}{n !}, k>0,
$$

is the solution of the linear second order differential equation of the form

$$
k z \omega^{\prime \prime}+(\gamma-k z) \omega^{\prime}-\beta \omega=0 .
$$

In this article, we call it confluent $k$-hypergeometric differential equation. It reduces to ordinary hypergeometric differential equation by taking $k \rightarrow 1$.

\section{Main Results}

Theorem 4.1. If $\lambda>0, \gamma>0$ and $x>t$, then

$$
\begin{aligned}
& \int_{t}^{x} \frac{(x-s)^{(\lambda / k)-1}}{\Gamma_{k}(\lambda)} \frac{(s-t)^{(\gamma / k)-1}}{\Gamma_{k}(\gamma)}{ }_{1} F_{1, k}\left[\begin{array}{l}
(\beta, k) ; \\
(\gamma, k) ;
\end{array}\right] \mathrm{d} s \\
& =\frac{k(x-t)^{((\gamma+\lambda) / k)-1}}{\Gamma_{k}(\gamma+\lambda)}{ }_{1} F_{1, k}\left[\begin{array}{c}
(\beta, k) ; \\
(\gamma+\lambda, k) ;
\end{array}\right]
\end{aligned}
$$

Proof. Consider

$$
\begin{aligned}
& \int_{0}^{1} \frac{(1-u)^{(\lambda / k)-1}}{\Gamma_{k}(\lambda)} \frac{u^{(\gamma / k)-1}}{\Gamma_{k}(\gamma)}{ }_{1} F_{1, k}\left[\begin{array}{l}
(\beta, k) ; \\
(\gamma, k) ;
\end{array}\right] \mathrm{d} u \\
& =\int_{0}^{1} \frac{(1-u)^{(\lambda / k)-1}}{\Gamma_{k}(\lambda)} \frac{u^{(\gamma / k)-1}}{\Gamma_{k}(\gamma)} \sum_{n=0}^{\infty} \frac{(\beta)_{n, k}}{(\gamma)_{n, k}} \frac{z^{n} u^{n}}{n !} \mathrm{d} u \\
& =k \sum_{n=0}^{\infty} \frac{(\beta)_{n, k}}{(\gamma)_{n, k}} \frac{z^{n}}{n !}\left\{\frac{1}{k} \int_{0}^{1} \frac{(1-u)^{(\lambda / k)-1}}{\Gamma_{k}(\lambda)} \frac{u^{(\gamma / k)+n-1}}{\Gamma_{k}(\gamma)} \mathrm{d} u\right\} \\
& =k \sum_{n=0}^{\infty} \frac{(\beta)_{n, k}}{(\gamma)_{n, k}} \frac{z^{n}}{n !} \frac{\Gamma_{k}(\gamma+n k)}{\Gamma_{k}(\gamma) \Gamma_{k}(\gamma+\lambda+n k)} \\
& =\frac{k}{\Gamma_{k}(\gamma+\lambda)} \sum_{n=0}^{\infty} \frac{(\beta)_{n, k}}{(\gamma+\lambda)_{n, k}} \frac{z^{n}}{n !} \\
& =\frac{k}{\Gamma_{k}(\gamma+\lambda)}{ }_{1} F_{1, k}\left[\begin{array}{c}
(\beta, k) ; \\
(\gamma+\lambda, k) ;
\end{array}\right]
\end{aligned}
$$

Put $u=\frac{s-t}{x-t}$ and $z=t-x$ in the above equation, then we get the desired result.

Theorem 4.2. Let

$$
\int_{0}^{x} \frac{(x-t)^{(\gamma / k)-1}}{\Gamma_{k}(\gamma)}{ }_{1} F_{1, k}\left[\begin{array}{l}
(\beta, k) ; \\
(\gamma, k) ;
\end{array} t-x\right] f(t) \mathrm{d} t=g(x)
$$

for $k>0, \beta>0, \gamma>0$ and $0<x<t<\infty$.

If $g(x)$ is a given function, then

$$
f(x)=\mathrm{e}^{-x} I_{k}^{-\beta} \mathrm{e}^{x} I_{k}^{-\gamma} I_{k}^{\beta} g(x) \text {. }
$$

Proof. Set

$$
\begin{aligned}
& H_{k}(\beta, \gamma) f(x) \\
& =\int_{0}^{x} \frac{(x-t)^{(\gamma / k)-1}}{\Gamma_{k}(\gamma)}{ }_{1} F_{1, k}\left[\begin{array}{l}
(\beta, k) ; \\
(\gamma, k) ;
\end{array} t-x\right] f(t) \mathrm{d} t,
\end{aligned}
$$

where $k>0, \beta>0, \gamma>0$ and $0<x<t<\infty$.

Apply $I_{k}^{\lambda}$ on both sides, we get the following

$$
\begin{aligned}
& I_{k}^{\lambda} H_{k}(\beta, \gamma) f(x)=\int_{0}^{x} \frac{(x-s)^{(\lambda / k)-1}}{\Gamma_{k}(\lambda)} \\
& \times\left\{\int_{0}^{s} \frac{(s-t)^{(\gamma / k)-1}}{\Gamma_{k}(\gamma)}{ }_{1} F_{1, k}\left[\begin{array}{l}
(\beta, k) ; t-s \\
(\gamma, k) ;
\end{array}\right] f(t) \mathrm{d} t\right\} \mathrm{d} s .
\end{aligned}
$$

Changing the order of integration by using Fubini's theorem.

$$
\begin{aligned}
& I_{k}^{\lambda} H_{k}(\beta, \gamma) f(x)=\int_{0}^{x} f(t) \\
& \times\left\{\int_{t}^{x} \frac{(x-s)^{(\lambda / k)-1}}{\Gamma_{k}(\lambda)} \frac{(s-t)^{(\gamma / k)-1}}{\Gamma_{k}(\gamma)}{ }_{1} F_{1, k}\left[\begin{array}{l}
(\beta, k) ; \\
(\gamma, k) ;
\end{array}\right] \mathrm{d} s\right\} \mathrm{d} t .
\end{aligned}
$$

By Theorem 4.1, we have

$$
\begin{aligned}
& I_{k}^{\lambda} H_{k}(\beta, \gamma) f(x) \\
& =k \int_{0}^{x} \frac{(x-t)^{((\gamma+\lambda) / k)-1}}{\Gamma_{k}(\gamma+\lambda)}{ }_{1} F_{1, k}\left[\begin{array}{r}
(\beta, k) ; \\
(\gamma+\lambda, k) ;
\end{array} \quad t-x\right] f(t) \mathrm{d} t .
\end{aligned}
$$

This implies that

$$
I_{k}^{\lambda} H_{k}(\beta, \gamma) f(x)=k H_{k}(\beta, \gamma+\lambda) f(x) .
$$

Since

$$
\begin{aligned}
& \int_{0}^{x} \frac{(x-t)^{(\gamma / k)-1}}{\Gamma_{k}(\gamma)}{ }_{1} F_{1, k}\left[\begin{array}{l}
(\beta, k) ; \\
(\gamma, k) ;
\end{array} t-x\right] f(t) \mathrm{d} t=g(x), \\
& H_{k}(\beta, \gamma) f(x)=g(x), \\
& I_{k}^{\beta} H_{k}(\beta, \gamma) f(x)=I_{k}^{\beta} g(x), \\
& k H_{k}(\beta, \beta+\gamma) f(x)=I_{k}^{\beta} g(x), \\
& I_{k}^{\gamma} H_{k}(\beta, \beta) f(x)=I_{k}^{\beta} g(x), \\
& H_{k}(\beta, \beta) f(x)=I_{k}^{-\gamma} I_{k}^{\beta} g(x) .
\end{aligned}
$$

This may be written as

$$
\begin{aligned}
& \int_{0}^{x} \frac{(x-t)^{(\beta / k)-1}}{\Gamma_{k}(\beta)}{ }_{1} F_{1, k}\left[\begin{array}{l}
(\beta, k) ; \\
(\beta, k) ;
\end{array} t-x\right] f(t) \mathrm{d} t \\
& =I_{k}^{-\gamma} I_{k}^{\beta} g(x) .
\end{aligned}
$$

Since ${ }_{1} F_{1, k}\left[\begin{array}{l}(\beta, k) ; \\ (\beta, k) ;\end{array} t-x\right]=\mathrm{e}^{t-x}$, we obtain 


$$
\begin{aligned}
& \int_{0}^{x} \frac{(x-t)^{(\beta / k)-1}}{\Gamma_{k}(\beta)} \mathrm{e}^{t-x} f(t) \mathrm{d} t=I_{k}^{-\gamma} I_{k}^{\beta} g(x), \\
& \int_{0}^{x} \frac{(x-t)^{(\beta / k)-1}}{\Gamma_{k}(\beta)} \mathrm{e}^{t} f(t) \mathrm{d} t=\mathrm{e}^{x} I_{k}^{-\gamma} I_{k}^{\beta} g(x), \\
& I_{k}^{\beta} \mathrm{e}^{x} f(x)=\mathrm{e}^{x} I_{k}^{-\gamma} I_{k}^{\beta} g(x), \\
& \mathrm{e}^{x} f(x)=I_{k}^{-\beta} \mathrm{e}^{x} I_{k}^{-\gamma} I_{k}^{\beta} g(x), \\
& f(x)=\mathrm{e}^{-x} I_{k}^{-\beta} \mathrm{e}^{x} I_{k}^{-\gamma} I_{k}^{\beta} g(x) .
\end{aligned}
$$

This is the solution of the integral equation, if it exists. This integral equation implies that

$$
g(x)=I_{k}^{-\beta} I_{k}^{\gamma} e^{-x} I_{k}^{\beta} e^{x} f(x) .
$$

Now, we find a solution of another integral equation

$$
\int_{0}^{x} \frac{(x-t)^{(\gamma / k)-1}}{\Gamma_{k}(\gamma)}{ }_{1} F_{1, k}\left[\begin{array}{l}
(\beta, k) ; x-t \\
(\gamma, k) ;
\end{array}\right] f(t) \mathrm{d} t=g(x),
$$

for $k>0, \beta>0, \gamma>0$ and $0<x<t<\infty$.

Theorem 4.3. Let

$$
\int_{0}^{x} \frac{(x-t)^{(\gamma / k)-1}}{\Gamma_{k}(\gamma)}{ }_{1} F_{1, k}\left[\begin{array}{l}
(\beta, k) ; x-t \\
(\gamma, k) ;
\end{array}\right] f(t) \mathrm{d} t=g(x),
$$

for $k>0, \beta>0, \gamma>0$ and $0<x<t<\infty$.

If $g(x)$ is a given function, then

$$
f(x)=I_{k}^{\beta-\gamma} \mathrm{e}^{x} I_{k}^{-\gamma} I_{k}^{\gamma-\beta} \mathrm{e}^{-x} g(x) .
$$

\section{Proof. Consider}

$$
\int_{0}^{x} \frac{(x-t)^{(\gamma / k)-1}}{\Gamma_{k}(\gamma)}{ }_{1} F_{1, k}\left[\begin{array}{l}
(\beta, k) ; x-t \\
(\gamma, k) ;
\end{array}\right] f(t) \mathrm{d} t=g(x) .
$$

Using the Mubeen's relation [15]

$$
{ }_{1} F_{1, k}\left[\begin{array}{l}
(\beta, k) ; \\
(\gamma, k) ;
\end{array}\right]=\mathrm{e}_{1}^{x} F_{1, k}\left[\begin{array}{r}
(\gamma-\beta, k) ; \\
(\beta, k) ;
\end{array}\right],
$$

we obtain the following

$$
\int_{0}^{x} \frac{(x-t)^{(\gamma / k)-1}}{\Gamma_{k}(\gamma)} \mathrm{e}^{x}{ }_{1} F_{1, k}\left[\begin{array}{r}
(\gamma-\beta, k) ; \\
(\gamma, k) ;
\end{array} \quad t-x\right] f(t) \mathrm{d} t=g(x) .
$$

Thus, if $k>0, \beta>0, \gamma>0$ and $0<x<t<\infty$, then

$$
f(x)=I_{k}^{\beta-\gamma} \mathrm{e}^{x} I_{k}^{-\gamma} I_{k}^{\gamma-\beta} \mathrm{e}^{-x} g(x) .
$$

Also, we have the following result

$$
g(x)=\mathrm{e}^{x} I_{k}^{\beta-\gamma} I_{k}^{\gamma} \mathrm{e}^{-x} I_{k}^{\gamma-\beta} f(x) .
$$

\section{Acknowledgements}

The author would like to express profound gratitude to referees for deeper review of this paper and their valuable advice and the referee's useful suggestions that led to an improved presentation of the paper. The author is also pleased to pay special thanks to Dr. Atiq ur Rehman for his support in this research.

\section{REFERENCES}

[1] S. Mubeen and G. M. Habibullah, " $k$-Fractional Integrals and Application," International Journal of Contemporary Mathematical Sciences, Vol. 7, No. 2, 2012, pp. 89-94.

[2] A. Erdélyi, "An Integral Equation Involving Legendre Functions," Journal of the Society for Industrial and Applied Mathematics, Vol. 12, No. 1, 1964, pp. 15-30. doi: $10.1137 / 0112002$

[3] E. R. Love, "Some Integral Equations Involving Hypergeometric Functions," Proceedings of the Edinburgh Mathematical Society, Vol. 15, No. 3, 1967, pp. 169-198. doi:10.1017/S0013091500011706

[4] G. M. Habibullah, "Some Integral Equations Involving Confluent Hypergeometric Functions," The Yokohama Mathematical Journal, Vol. 19, 1971, pp. 35-43.

[5] K. N. Srivastava, "Fractional Integration and Integral Equations with Polynomial Kernels," Journal of the Society for Industrial and Applied Mathematics, Vol. 40, 1965, pp. 435-440.

[6] R. Diaz and C. Teruel, " $q, k-$ Generalized Gamma and Beta Functions," Journal of Nonlinear Mathematical Physics, Vol. 12, No. 1, 2005, pp. 118-134. doi:10.2991/jnmp.2005.12.1.10

[7] R. Diaz and E. Pariguan, "On Hypergeometric Functions and Pochhammer k-Symbol," Divulgaciones Matemáticas, Vol. 15, No. 2, 2007, pp. 179-192.

[8] R. Diaz, C. Ortiz and E. Pariguan, "On the k-Gamma q-Distribution," Central European Journal of Mathematics, Vol. 8, No. 3, 2010, pp. 448-458. doi:10.2478/s11533-010-0029-0

[9] M. Mansour, "Determining the $k$-Generalized Gamma Function $\Gamma_{k}(x)$ by Functional Equations," International Journal of Contemporary Mathematical Sciences, Vol. 4, No. 21, 2009, pp. 1037-1042.

[10] C. G. Kokologiannaki, "Properties and Inequalities of Generalized $k$-Gamma, Beta and Zeta Functions," International Journal of Contemporary Mathematical Sciences, Vol. 5, No. 13-16, 2010, pp. 653-660.

[11] V. Krasniqi, "A Limit for the $k$-Gamma and $k$-Beta Function," International Mathematical Forum, Vol. 5, No. 33 , 2010, pp. 1613-1617.

[12] V. Krasniqi, "Inequalities and Monotonicity for the Ration of k-Gamma Function," Scientia Magna, Vol. 6, No. 1, 2010, pp. 40-45.

[13] F. Merovci, "Power Product Inequalities for the $\Gamma_{k}$ Function," International Journal of Mathematical Analysis, Vol. 4, No. 21-24, 2010, pp. 1007-1012.

[14] S. Mubeen and G. M. Habibullah, "An Integral Representation of Some k-Hypergeometric Functions," International Mathematical Forum, Vol. 7, No. 4, 2012, pp. 203-207.

[15] S. Mubeen, "k-Analogue of Kummer's First Formula," Journal of Inequalities and Special Functions, Vol. 3, No. 3, 2012, pp. 41-44. 